\section{Bicarbonate Level for Early Diagnosis of Obesity Hypoventilation Syndrome: Still Defining Boundaries}

\section{To the Editor:}

Given the current epidemic of obesity, it is likely that obesity hypoventilation syndrome (OHS), the form of chronic respiratory failure specifically attributable to obesity, is becoming the second leading cause of chronic respiratory insufficiency worldwide after COPD. ${ }^{1}$ As opposed to the latter, no large public health campaign has yet been conducted to improve the diagnostic process of OHS, and the disease is largely underrecognized, usually discovered at an advanced stage when acute respiratory failure occurs. ${ }^{2}$ In their prospective observational study, Bingol et $\mathrm{al}^{3}$ compare the clinical characteristics and polysomnographic parameters of OHS subjects with those of obese subjects with pure OSAS. Finding that bicarbonate level in plasma was higher and the nadir $\mathrm{S}_{\mathrm{pO}_{2}}$ at night was lower in the OHS group compared with the obstructive obesity sleep apnea syndrome group, they concluded that these parameters can help in improving the diagnosis of OHS in specialized sleep medicine centers without the use of an arterial puncture to obtain arterial blood gas analysis. ${ }^{4}$ We think that, although attractive for specialists in sleep medicine, this study missed its target. What is interesting is that the present study confirms the opinion of a panel of international experts regarding the diagnostic value of bicarbonate level to assess OHS in obese snorers. ${ }^{5}$ However, the diagnosis of OHS is not a difficult issue reserved for specialists in sleep medicine. In this line, some key practical aspects need to be taken into account in this study. First, it is largely underdetected by general practitioners, and only a minority of the numerous obese patients at risk are referred early to a specialized center for a polygraphic sleep study. Second, even when admitted to the ICU for an episode of acute respiratory failure, up to $75 \%$ of the morbidly obese patients with OHS are misdiagnosed as having COPD or asthma. ${ }^{3}$ So the point is not to prevent a puncture of the radial artery to make the diagnosis less invasive in specialized sleep centers; this seems from a practical point of view crucial when some respiratory diseases like pulmonary hypertension require a pulmonary arterial catheter for assessment. Furthermore, these patients will continue to have many arterial punctures in the ICU and sometimes an endotracheal tube as a bonus if we do not improve the early diagnostic process and management of the disease. Manuel et $\mathrm{al}^{5}$ have recently described a new phenotype of obese subjects with high bicarbonate level $(>27 \mathrm{mmol} / \mathrm{L})$ and normal $\mathrm{P}_{\mathrm{aCO}_{2}}(<45 \mathrm{~mm} \mathrm{Hg})$ that can be considered as developing an incipient form of OHS. Determining bicarbonate level in obese patients at risk may improve the early detection of OHS by general practitioners, who can then refer these patients to a pulmonologist early in the course of the disease. Finally, the therapeutic goals include facilitating the use of noninvasive ventilation and extending its practice and always treating OHS as one of the multiple disorders associated with obesity.

Malcolm Lemyze MD

Department of Respiratory and Critical Care Medicine Schaffner Hospital Lens, France

Antonio M Esquinas MD PhD Intensive Care Unit Hospital Morales Meseguer Murcia, Spain

The authors have disclosed no conflicts of interest.

DOI: $10.4187 /$ respcare. 04260

\section{REFERENCES}

1. Finucane MM, Stevens GA, Cowan MJ, Danaei G, Lin JK, Paciorek CJ, et al. National, regional, and global trends in bodymass index since 1980: systematic analysis of health examination surveys and epidemiological studies with 960 country-years and 9.1 million participants. Lancet 2011; 377(9765):557-567.

2. Quint JK, Ward L, Davison AG. Previously undiagnosed obesity hypoventilation syndrome. Thorax 2007;62(5):462-463.

3. Bingol Z, Pihtılı A, Cagatay P, Okumus G, Kıyan E. Clinical predictors of obesity hypoventilation syndrome in obese subjects with obstructive sleep apnea. Respir Care 2015;60(5):666-672.

4. Hart N, Mandal S, Manuel A, Mokhlesi B, Pépin JL, Piper A, Stradling JR. Obesity hypoventilation syndrome: does the current definition need revisiting? Thorax 2014; 69(1):83-84.

5. Manuel AR, Hart N, Stradling JR. Is a raised bicarbonate, without hypercapnia, part of the physiological spectrum of obesity-related hypoventilation? Chest 2015;147(2): 362-368.

\section{Bicarbonate Level for Early Diagnosis of Obesity Hypoventilation Syndrome: Still Defining Boundaries-Reply}

\section{In reply:}

We thank Drs Lemyze and Esquinas for their interest in our work. ${ }^{1}$ The International
Classification of Sleep Disorders, 3rd edition, ${ }^{2}$ defines obesity hypoventilation syndrome (OHS) as the combined presence of obesity (body mass index $>30 \mathrm{~kg} / \mathrm{m}^{2}$ ) with awake arterial hypercapnia $\left(\mathrm{P}_{\mathrm{aCO}_{2}}>45 \mathrm{~mm} \mathrm{Hg}\right)$ and sleep-disordered breathing in the absence of other causes of alveolar hypoventilation, such as COPD. It is an important cause of chronic respiratory failure. ${ }^{3}$ Therefore, it is very important to raise awareness of this disease. As you mentioned, these patients are frequently misdiagnosed in outpatient clinics, primary care and intensive care units as having COPD or asthma., ${ }^{4,5}$ OHS diagnosis is underestimated, and most OHS patients are given bronchodilator and long-term oxygen therapy. Additionally, most obese patients are discharged from the emergency department and ICUs without long-term noninvasive mechanical ventilation, with only $30 \%$ of hospitalized patients receiving a correct diagnosis of OHS when admitted with hypercapnic respiratory failure. ${ }^{4,6}$ Generally, the diagnosis of OHS as the cause of respiratory failure is not appreciated until referral to a respiratory physician has been made.

Nearly $90 \%$ of OHS patients have coexisting obstructive sleep apnea (OSA) ${ }^{7,8}$ Obese patients are mostly referred to the sleep laboratory due to suspicion of OSA. Another problem is that OHS has been incorrectly considered to be a severe form of OSA. ${ }^{9}$ Although the diagnosis of OHS may not be difficult for sleep specialists and chest physicians, the exact OHS frequency is still not known because capnography and arterial blood gas analysis, which are required for diagnosis, are not routinely performed in sleep laboratories. This may result in inadequate treatment. Because some OHS patients do not benefit from CPAP, bi-level positive airway pressure may be a better choice.

In light of these data, we aimed to improve the awareness of OHS with our article. For this aim, we tried to highlight the differences between OHS and pure OSA in obese subjects. We selected individuals who were referred to the sleep laboratory because we would have the chance to find more OHS subjects. We thought that finding the differences between OHS and pure OSA would improve the diagnostic process. Having higher serum bicarbonate level is one of the differences between OHS and OSA. Although arterial blood gas is not a very invasive procedure, more practical less invasive or noninvasive methods will im- 